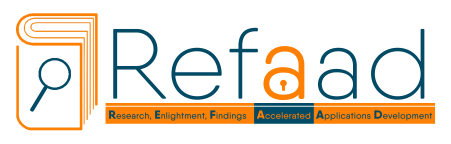

General Letters in Mathematics Vol. 2, No. 3, June 2017, pp.105-110

e-ISSN 2519-9277, p-ISSN 2519-9269

Available online at http:// www.refaad.com

\title{
Oscillatory Behavior of Higher-Order Delay Differential Equations
}

\section{O. Bazighifan}

Department of Mathematics, Hadhramout University, Yemen.

○.bazighifan@gmail.com

Abstract. This paper is concerned with asymptotic and oscillatory properties of the nonlinear higher-order differential equation with delay argument. Some examples are given to illustrate our main results .

Keywords: Oscillation, higher-order, delay differential equations.

\section{Introduction and preliminaries}

In this work, we study the oscillation of higher-order delay differential equation

$$
\left[b(t)\left(x^{(n-1)}(t)\right)^{\gamma}\right]^{\prime}+q(t) f(x(\tau(t)))=0 \quad t \geq t_{0}
$$

We assume that the following assumptions hold:

$\left(H_{1}\right) \gamma$ is a quotient of odd positive integers;

$\left(H_{2}\right) \quad b \in C^{1}\left[t_{0} \infty\right), b^{\prime}(t) \geq 0, b(t)>0, q, \tau \in C\left[t_{0} \infty\right), f \in C(\mathbb{R} \mathbb{R})$, and $-f(-x y) \geq f(x y) \geq f(x) f(y)$, for $x y>0, q>0, \tau(t) \leq t, \lim _{t \rightarrow \infty} \tau(t)=\infty$.

(H3) there exist constants $k>0$ such that $f(u) / u^{\gamma} \geq k$, for $u \neq 0$.

By a solution of Eq. (1.1) we mean a function $x \in C^{(n-1)}\left[T_{x} \infty\right), T_{x} \geq t_{0}$, which has the property $b(t)\left(x^{(n-1)}(t)\right)^{\gamma}$

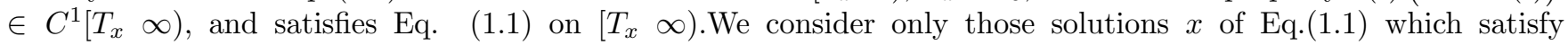
$\sup \{|x(t)|: t \geq T\}>0$, for all $T>T_{x}$. We assume that (1.1) possesses such a solution. A solution of (1.1) is called oscillatory if it has arbitrarily large zeros on $\left[T_{x} \infty\right)$, and otherwise, it is called to be nonoscillatory. (1.1) is said to be oscillatory if all its solutions are oscillatory. In what follows, we present some related results that served as a motivation for the contents of this paper.

E.M.Elabbasy, et al. 9] study the a symptotic properties and oscillation of the nth-order delay differential equation

$$
\left[r(t)\left(x^{(n-1)}(t)\right)^{\gamma}\right]^{\prime}+\sum_{i=1}^{m} q_{i}(t) g\left(x\left(\tau_{i}(t)\right)\right)=0 \quad t \geq t_{0} .
$$

C.Zhang, et al. 20] and Zhang, et al. 21] consider the oscillatory properties of the higher-order differential equation

$$
\left[b(t)\left(x^{(n-1)}(t)\right)^{\gamma}\right]^{\prime}+q(t) x^{\gamma}(\tau(t))=0 \quad t \geq t_{0} .
$$

under the conditions

$$
\int_{t_{\circ}}^{\infty} \frac{1}{b^{\frac{1}{\gamma}}(t)} d t=\infty
$$


and

$$
\int_{t_{\circ}}^{\infty} \frac{1}{b^{\frac{1}{\gamma}}(t)} d \dot{t}<\infty
$$

Our aim in the present paper is to employ the Riccatti technique to establish some conditions for the oscillation of all solutions of (1.1). Some examples are presented to illustrate our main results.

\section{Main Results}

In this section, we shall establish some oscillation criteria for (1.1). We begin with the following lemma.

Lemma 2.1. Let $z \in\left(C^{n}\left[t_{0} \infty\right], \mathbb{R}^{+}\right)$and assume that $z^{(n)}$ is of fixed sign and not identically zero on a subray of $\left[t_{0} \infty\right]$. If, moreover, $z(t)>0, z^{(n-1)}(t) z^{(n)}(t) \leq 0$ and $\lim _{t \rightarrow \infty} z(t) \neq 0$, then, for every $\lambda \in(0,1)$, there exists $t_{\lambda} \geq t_{\circ}$ such that

$$
z(t) \geq \frac{\lambda}{(n-1) !} t^{n-1}\left|z^{\left({ }^{n-1}\right)}(t)\right|, \text { for } t \in\left[t_{\lambda}, \infty\right) .
$$

We are now ready to state and prove the main results. For convenience, we denote

$$
\begin{aligned}
& \pi(s):=\int_{t_{\circ}}^{\infty} \frac{1}{b^{\frac{1}{\gamma}}(s)} d s, \delta^{\prime}(t):=\max \left\{0, \delta^{\prime}(t)\right\}, \\
& \sigma(t)=\frac{\int_{t}^{\infty}(\mu-t)^{(n-4)} \pi(\mu) d \mu}{(n-4) !} .
\end{aligned}
$$

Theorem 2.2. Let $n \geq 4$. Assume that (1.3) holds. Further, assume that for some constant $\lambda \in(0,1)$, the differential equation

$$
y^{\prime}(t)+q(t) f\left(\frac{\lambda}{(n-1) ! b^{1 / \gamma}(\tau(t))} \tau^{n-1}(t)\right) f\left(y^{1 / \gamma}(\tau(t))\right)=0,
$$

is oscillatory. If

$$
\lim _{t \rightarrow \infty} \sup _{t_{\circ}}\left[k q(t)\left(\frac{\tau^{3}(t)}{t^{3}}\right)^{\gamma} \delta(s)-\frac{\left(\delta^{\prime}(s)\right)^{\gamma+1}}{(\gamma+1)^{\gamma+1}(\delta(s))^{2 \gamma+1} \sigma^{\gamma}(s)}\right] d s=\infty,
$$

holds. Then every solution of (1.1) is oscillatory.

Proof. Assume that (1.1) has a nonoscillatory solution $x$. Without loss of generality, we can assume that $x(t)>0$. It follows from (1.1) that there exist two possible cases:

case1 : $x^{(n-1)}(t)>0, x^{(n)}(t)<0,\left(b\left(x^{(n-1)}\right)^{\gamma}\right)^{\prime}(t) \leq 0$.

case $2: x^{(n-2)}(t)>0, x^{(n-1)}(t)<0,\left(b\left(x^{(n-1)}\right)^{\gamma}\right)^{\prime}(t) \leq 0$.

for $t>t_{1}, t_{1}$ is large enough.

Assume that case(1) holds. From Lemma 2.1, we have

$$
x(\tau(t)) \geq \frac{\lambda \tau^{n-1}(t)}{(n-1) ! b^{1 / \gamma}(t)}\left(b^{1 / \gamma}(t) x^{(n-1)} \tau(t)\right),
$$

for every $\lambda \in(0,1)$. Using (2.3) in Eq. (1.1), we see that

$y(t)=b(t)\left[x^{(n-1)}(t)\right]^{\gamma}$ is a positive solution of the differential inequality

$$
y^{\prime}(t)+q(t) f\left(\frac{\lambda}{(n-1) ! b^{1 / \gamma}(\tau(t))} \tau^{n-1}(t)\right) f\left(y^{1 / \gamma}(\tau(t))\right) \leq 0,
$$

By Theorem 1 in [18, we conclude that the corresponding equation (1.1) also has a positive solution. This contradiction.

Assume that case (2) holds. Noting that $b(t)\left(x^{(n-1)}(t)\right)^{\gamma}$ is decreasing, we obtain

$$
\begin{aligned}
b^{\frac{1}{\gamma}}(s) x^{(n-1)}(s) & \leq b^{\frac{1}{\gamma}}(t) x^{(n-1)}(t), s \geq t \geq t_{1}, \\
x^{(n-1)}(s) & \leq b^{\frac{1}{\gamma}}(t) x^{(n-1)}(t) b^{\frac{-1}{\gamma}}(s) .
\end{aligned}
$$


Integrating again from $t$ to $v$, we get

$$
x^{(n-2)}(t)-x^{(n-2)}(v) \geq-b^{\frac{1}{\gamma}}(t) x^{(n-1)}(t) \int_{t}^{v} b^{\frac{-1}{\gamma}}(s) d s .
$$

Letting $v \rightarrow \infty$; we obtain

$$
x^{(n-2)}(t) \geq-b^{\frac{1}{\gamma}}(t) x^{(n-1)}(t) \pi(t) .
$$

Integrating from $t$ to $\infty$, we get

$$
-x^{(n-3)}(t) \geq-b^{\frac{1}{\gamma}}(t) x^{(n-1)}(t) \int_{t}^{\infty} \pi(s) d s .
$$

Similarly, integrating the above inequality from $t$ to $\infty$ a total of $(n-4)$ times, we find

$$
-x^{\prime}(t) \geq \frac{-b^{\frac{1}{\gamma}}(t) x^{(n-1)}(t)}{(n-4) !} \int_{t}^{\infty}(\mu-t)^{(n-4)} \pi(\mu) d \mu .
$$

Define the function $\omega(t)$ by

$$
\omega(t):=\delta(t) \frac{b(t)\left(x^{(n-1)}\right)^{\gamma}(t)}{(x(t))^{\gamma}} .
$$

Then $\omega(t)<0$ for $t \geq t_{1}$ and

$$
\begin{aligned}
\omega^{\prime}(t)= & \delta^{\prime}(t) \frac{b(t)\left(x^{(n-1)}\right)^{\gamma}(t)}{(x(t))^{\gamma}}+\delta(t) \frac{\left(b\left(x^{(n-1)}\right)^{\gamma}\right)^{\prime}(t)}{(x(t))^{\gamma}} \\
& -\gamma \delta(t) \frac{(x)^{\prime}(t) b(t)\left(x^{(n-1)}\right)^{\gamma}(t)}{(x)^{\gamma+1}(t)}
\end{aligned}
$$

By the Kiguradze, we find $x(t) \geq(t / 3) x^{\prime}(t)$ and, hence

$$
\frac{x\left(\tau_{i}(t)\right)}{x(t)} \geq \frac{\tau_{i}^{3}(t)}{t^{3}} .
$$

It follows from (1.1) and (2.6), we get

$$
\begin{aligned}
\omega^{\prime}(t) \leq & -k q(t)\left(\frac{\tau^{3}(t)}{t^{3}}\right)^{\gamma} \delta(t)+\frac{\delta^{\prime}(t)}{\delta(t)} \omega(t) \\
& -\gamma \delta(t) \frac{\int_{t}^{\infty}(\mu-t)^{(n-4)} \pi(\mu) d \mu}{(n-4) !} \omega^{\frac{\gamma+1}{\gamma}}(t) . \\
\omega^{\prime}(t) \leq & -k q(t)\left(\frac{\tau^{3}(t)}{t^{3}}\right)^{\gamma} \delta(t)+\frac{\delta^{\prime}(t)}{\delta(t)} \omega(t) \\
& -\gamma \delta(t) \sigma(t) \omega^{\frac{\gamma+1}{\gamma}}(t) .
\end{aligned}
$$

Define now

$$
C:=\gamma \delta(t) \sigma(t), D:=\frac{\delta^{\prime}(t)}{\delta(t)}, y:=\omega(t)
$$

Appling the inequality

$$
D y-C y^{\frac{\gamma+1}{\gamma}} \leq \frac{\gamma^{\gamma}}{(\gamma+1)^{\gamma+1}} \frac{D^{\gamma+1}}{C^{\gamma}}, C, D>0
$$

we find

$$
\frac{\delta^{\prime}(t)}{\delta(t)} \omega(t)-\gamma \delta(t) \sigma(t) \omega(t)^{\frac{\gamma+1}{\gamma}} \leq \frac{\left(\delta^{\prime}(t)\right)^{\gamma+1}}{(\gamma+1)^{\gamma+1}(\delta(t))^{2 \gamma+1} \sigma^{\gamma}(t)}
$$


Hence, we obtain

$$
\omega^{\prime}(t) \leq-k q(t)\left(\frac{\tau^{3}(t)}{t^{3}}\right)^{\gamma} \delta(t)-\frac{\left(\delta^{\prime}(t)\right)^{\gamma+1}}{(\gamma+1)^{\gamma+1}(\delta(t))^{2 \gamma+1} \sigma^{\gamma}(t)} .
$$

Integrating from $t_{1}$ to $t$, we get

$$
{ }_{t_{1}}^{t}\left[k q(s)\left(\frac{\tau^{3}(s)}{s^{3}}\right)^{\gamma} \delta(s)-\frac{\left(\delta^{\prime}(s)\right)^{\gamma+1}}{(\gamma+1)^{\gamma+1}(\delta(s))^{2 \gamma+1} \sigma^{\gamma}(s)}\right] d s \leq \omega\left(t_{1}\right),
$$

which contradicts $(2.2)$.

Theorem 2.1 is proved.

Corollary 2.3. Let $n \geq 4$. Assume that (1.3) holds. If

$$
\lim _{t \rightarrow \infty} \inf \int_{\tau(t)}^{\infty} q(s) f\left(\frac{\lambda}{(n-1) ! b^{1 / \gamma}(\tau(s))} \tau^{n-1}(s)\right) d s>\frac{((n-1) !)^{\gamma}}{e},
$$

and

$$
\lim _{t \rightarrow \infty} \sup _{t_{\circ}}\left[k q(s)\left(\frac{\tau^{3}(s)}{s^{3}}\right)^{\gamma} \delta(s)-\frac{\left(\delta^{\prime}(s)\right)^{\gamma+1}}{(\gamma+1)^{\gamma+1}(\delta(s))^{2 \gamma+1} \sigma^{\gamma}(s)}\right] d s=\infty,
$$

holds for some constant $\lambda \in(0,1)$. Then every solution of is oscillatory or tends to zero. If

Corollary 2.4. Let $n \geq 4$. Assume that (1.3) holds. and let $\beta$ be the ratio of two odd positive integers with $\beta<\gamma$.

$$
\lim _{t \rightarrow \infty} \sup \int_{\tau(t)}^{\infty} q(s) \frac{\left(\tau^{n-1}(s)\right)^{\beta}}{b^{\frac{\beta}{\gamma}}(\tau(s))}>0
$$

then the differential equation

$$
\left[r(t)\left(x^{(n-1)}(t)\right)^{\gamma}\right]^{\prime}+q(t) x^{\beta}(\tau(t))=0 \quad t \geq t_{\circ}
$$

is oscillatory.

\section{$3 \quad$ Examples}

We give the following example to illustrate our main results.

Consider a differential equation

$$
\left(t^{6}\left(x^{\prime \prime \prime}(t)\right)^{3}\right)^{\prime}+\frac{\beta+t^{2}+t}{t} x^{3}\left(\frac{t}{2}\right)=0, t \geq 1
$$

where $\beta>0$ is a constant. Let

$$
\begin{aligned}
\gamma & =3, n=4, b(t)=t^{6}>0, b^{\prime}(t)=6 t^{5} \geq 0, b \in C^{1}\left[t_{\circ} \infty\right) \\
q(t) & =\frac{\beta+t^{2}+t}{t}>0, q \in C\left[t_{\circ} \infty\right) \\
\tau(t) & =\frac{t}{2} \leq t, \lim _{t \rightarrow \infty} \frac{t}{2}=\infty, \tau(t) \in C\left[t_{\circ} \infty\right)
\end{aligned}
$$

we see $\left(H_{1}\right),\left(H_{2}\right)$ and $\left(H_{3}\right)$ holds. Then, we find

$$
\pi(s):=\int_{t}^{\infty} \frac{1}{b^{\frac{1}{\gamma}}(s)} d s=\int_{t}^{\infty} \frac{1}{\left(s^{6}\right)^{\frac{1}{3}}} d s=\frac{1}{t},
$$

we now set $\delta(s)=1$, It is easy to see that all conditions of Corollary 2.1 hold. Hence every solution of (3.1) is oscillatory. 
Example 3.1. Consider a differential equation

$$
\left(t^{3}\left(x^{\prime \prime \prime}(t)\right)\right)^{\prime}+\frac{1}{t}\left(x^{2}+x\right)(\alpha t)=0, t \geq 1,
$$

where $0<\alpha<1$ is a constant. Let

$$
\begin{aligned}
\gamma & =1, n=4, b(t)=t^{3}>0, b^{\prime}(t)=9 t^{8} \geq 0, b \in C^{1}\left[t_{0} \infty\right), \\
q(t) & =\frac{1}{t}>0, q \in C\left[t_{0} \infty\right), \\
\tau(t) & =\alpha t \leq t, \lim \alpha t=\infty, \tau(t) \in C\left[t_{0} \infty\right),
\end{aligned}
$$

we see $\left(H_{1}\right),\left(H_{2}\right)$ and $\left(H_{3}\right)$ holds. Then, we find

$$
\pi(s):=\int_{t}^{\infty} \frac{1}{b^{\frac{1}{\gamma}}(s)} d s=\int_{t}^{\infty} \frac{1}{s^{3}} d s=\frac{1}{2 s^{2}}
$$

IF we now set $\delta(s)=1$. It is easy to see that all conditions of Corollary 2.1 hold. Hence every solution of (3.2) is oscillatory. However, theresults of [21] cannot confirm this conclusion.

Example 3.2. Consider a differential equation

$$
\left(e^{t} x^{\prime \prime \prime}(t)\right)^{\prime}+2 \sqrt{10} e^{\arcsin \frac{\sqrt{10}}{10}+t} x\left(t-\arcsin \frac{\sqrt{10}}{10}\right)=0,
$$

$$
\begin{aligned}
\text { Let } \gamma & =1, n=4, b(t)=e^{t}>0, b^{\prime}(t)=e^{t} \geq 0, b \in C^{1}\left[t_{0} \infty\right] . \\
q(t) & =2 \sqrt{10} e^{\arcsin \frac{\sqrt{10}}{10}+t}, q \in C\left[t_{0} \infty\right] . \\
\tau(t) & =t-\arcsin \frac{\sqrt{10}}{10} \leq t, \lim _{t \rightarrow \infty} t-\arcsin \frac{\sqrt{10}}{10}=\infty, \tau(t) \in C\left[\begin{array}{ll}
t_{0} \infty
\end{array}\right],
\end{aligned}
$$

we see $\left(H_{1}\right),\left(H_{2}\right)$ and $\left(H_{3}\right)$ holds. Then, we get

$$
\begin{aligned}
& \pi(s) \quad: \quad=\int_{t}^{\infty} \frac{1}{b^{\frac{1}{\gamma}}(s)} d s=\int_{t}^{\infty} \frac{1}{\left(e^{s}\right)} d s=e^{-t} \\
& \sigma(t)=\frac{\int_{t}^{\infty}(\mu-t)^{(n-4)} \pi(\mu) d \mu}{(n-4) !}=\int_{t}^{\infty} e^{-s} d s=e^{-t}
\end{aligned}
$$

IF we now set $\delta(s)=1$,we get

$$
\lim _{t \rightarrow \infty} \sup _{t_{\circ}}\left[k q(s)\left(\frac{\tau^{3}(s)}{s^{3}}\right)^{\gamma} \delta(s)-\frac{\left(\delta^{\prime}(s)\right)^{\gamma+1}}{(\gamma+1)^{\gamma+1}(\delta(s))^{2 \gamma+1} \sigma^{\gamma}(s)}\right] d s=\infty .
$$

Then we can easily see that all assumptions of Corollary 2.1 are satisfied. Hence (3.3) is oscillatory.

\section{References}

[1] R.P.Agarwal, S.R.Grace And D.O'Regan, The oscillation of certain higher-order functional differential equations, Math.Comput.Model., 37,705-728(2003).

[2] R.P.Agarwal, S.R.Grace and D.O'Regan, Oscillation criteria for certain $\mathrm{n}$ th order differential equations with deviating arguments, J.Math.Anal.Appl., 262,601-622(2001).

[3] B. Baculikova, J. Dzurina, J.R.Graef, On the oscillation of higher-order delay differential equations, Math. Slovaca 187 (4) (2012) $387-400$ 
[4] B. Baculikova, E. M. ElabbasyAnd S. H. Saker, Oscillation criteria for third-order nonlinear differential equations, Math. Slovaca 58 (2) (2008) 1 - 20.Zbl pre05255054.

[5] B. Baculikova And J. Dzurina, Oscillation of third-order nonlinear differential equations, Appl .Math.Lett. , $24,466-470(2011)$.

[6] E.M.Elabbasy, M.Y.Barsoum And O.Moaaz, Boundedness and oscillation of third order neutral differential equations with deviating arguments, Journal of Applied Mathematics and Physics, 3, 1367-1375(2015).

[7] E.M.Elabbasy And O.Moaaz, On the Oscillation of Third Order Neutral Differential Equations , Asian Journal of Mathematics and Applications.274, 1-11(2016).

[8] E.M.Elabbasy And O.Moaaz, On the asymptotic behavior of third-order nonlinear functional differential equations , Serdica Mathematical Journal 42, 157-174(2016).

[9] E. M. Elabbasy, O. Moaaz, O. Bazighifan, Oscillation Solution for Higher-Order Delay Differential Equations, Journal of King Abdulaziz University. 29, p7(2017).

[10] O. Moaaz, E. M. Elabbasy, O. Bazighifan, On the asymptotic behavior of fourth-order functional differential equations, Advances in Difference Equations. 261, 1-13(2017).

[11] E. M. Elabbasy, O. Moaaz, O. Bazighifan, Oscillation of Fourth-Order Advanced Differential Equations, Journal of Modern Science \& Engineering. 3, 64-71(2017).

[12] E. M. Elabbasy, O. Moaaz, O. Bazighifan, Oscillation Criteria for Fourth-Order Delay Differential Equations, International Journal of Modern Mathematical Sciences. 15, 50-57(2017).

[13] L.H.Erbe, Q.Kong And B.G.Zhang, Oscillation theory for functional differential equations, Marcel Dekker, New York, 1994, Zbl0821. 34067.

[14] S.R.Grace, R.P.Agarwal And J.R.Graef, Oscillation theorems for fourth order functional differential equations, J.Appl.Math.Comput., 30,75-88(2009)..

[15] I.Gyori, G. Ladas, Oscillation theory of delay differential equations with applications, Oxford (1991).

[16] G.S.Ladde, V.Lakshmikantham And B.G.Zhang, Oscillation theory of differential equations with deviating arguments, Marcel Dekker,NewYork,(1987)

[17] Ch.G.Philos, A new criteri on forthe oscillatory and asymptotic behavior of delay differential equations, Bull. A cad. Pol. Sci., S' er .Sci. Math., 39,61-64(1981).

[18] C.G.Philos, On the existence of nonoscillatory solutions tending to zero at $\infty$ for differential equations with positive delay, Arch. Math. (Basel), 36,168-178(1981).

[19] S.H.Saker, Oscillation theory of delay differential and difference equations.Second and third orders, Verlag Dr M“* uller,Germany(2010).

[20] C.Zhang, R.P.Agarwal, M.Bohner And T.Li, New results for oscillatory behaviorof even-order half-linear delay differential equations , Appl .Math .Lett., 26,179-183(2013).

[21] C.Zhang, T.Li, B.Sun And E.Thandapani, On the oscillation of higher-order half-linear delay differential equations, Appl .Math .Lett., 24,1618-1621(2011).

[22] C.Zhang, T.Li And S.H.Saker, Oscillation of fourth-order delay differential quations, Journal of Mathematical Sciences, $201,296-308(2014)$. 